\title{
Orientation-specificity in kinesthetic spatial learning: The role of multiple orientations
}

\author{
CLARK C. PRESSON and NINA DELANGE \\ Arizona State University, Tempe, Arizona \\ and \\ MARK D. HAZELRIGG \\ University of Missouri, Columbia, Missouri
}

\begin{abstract}
In the current study we tested whether multiple orientations in kinesthetic learning affected how flexibly spatial information is stored and later used in making location judgments. Three groups learned simple routes by walking them while blindfolded, with (1) multiple orientations achieved through normal walking, (2) multiple orientations achieved through backward walking, or (3) a single orientation achieved through walking without turning (which required forward, backward, and sideways walking). When subjects had experienced multiple orientations while learning the routes, later directional judgments were equally accurate (and equally rapid) regardless of whether the judgments were aligned or were contra-aligned with the orientation of the routes as originally learned. In contrast, when routes were learned in a single orientation (without turning), subsequent judgments on contra-aligned trials were both less accurate and slower than judgments on aligned trials. Thus, multiple orientations are important to establish orientation-free, flexible use of spatial information in a kinesthetic learning environment. This contrasts with the pattern of results typically found in visual spatial learning and suggests that the factors that affect orientation specificity of spatial use may differ across spatial modality.
\end{abstract}

In recent years, there has been a growing interest in understanding the nature of spatial representations and the manner in which spatial knowledge is used (e.g., Liben, 1981; Mandler, 1983). One issue has been whether spatial information is coded in an orientation-specific manner (easily used only in the orientation encountered during learning) or whether it is orientation free (coded in such a way as to be available in several orientations regardless of initial learning). Empirical work on orientation specificity of spatial coding has identified examples of both types of coding in object perception (Jolicoeur \& Kosslyn, 1983), as well as in large-scale environments (e.g., Evans \& Pezdek, 1980).

Levine, Jankovic, and Palij (1982) argued that spatial knowledge generally is stored in a specific orientation, like a physical map. They demonstrated that when people learn spatial information from a map (or other symbolic representation), it is stored and recalled in the same specific orientation as that initially learned (see also Evans \& Pezdek, 1980; Presson \& Hazelrigg, 1984). This orientation specificity biases later judgments that rely on the map information: Judgments are easy if subjects are aligned to the information the same way they had learned

This research was supported in part by NIH Research Grant HD 20666 to the first author. The authors thank David Martinez, Michael Hanson, and the Spatial Cognition Research Group for assistance in running subjects, and Laurie Chassin for helpful comments. Address requests for reprints to Clark C. Presson, Department of Psychology, Arizona State University, Tempe, AZ 85287. it on the map, and are more difficult if subjects are contraaligned to the information as learned.

Although map information consistently shows orientationspecific effects, some types of spatial information are not always used in orientation-specific ways. When equivalent spatial information is obtained through navigation, the information is represented in a more orientation-free manner. In these cases, people are not biased to recall and use the information in a single orientation, and judgments are made equally easily regardless of the alignment of the person when making a judgment. This finding has been shown for sighted navigation of large-scale environments (Evans \& Pezdek, 1980; Thorndyke \& Hayes-Roth, 1982), as well as for nonsighted navigation of simple routes (Presson \& Hazelrigg, 1984). Evans and Pezdek (1980) suggested that one of the critical factors leading subjects to code the information in more flexible ways is that navigation typically entails multiple vantage points during learning. For both sighted and nonsighted walking, a person normally turns and experiences different orientations toward the information while it is being initially integrated. These multiple orientations while walking may be critical to the establishment of the orientationfree coding shown previously for kinesthetic learning conditions, although this has never been tested directly.

The current study explicitly tested the role of multiple orientations when spatial learning was achieved with (nonsighted) kinesthetic locomotion. Three groups learned simple routes by walking them while blindfolded. Turn- 
ing as appropriate for each segment of the route, the subjects in two of these groups experienced the routes from multiple orientations. One group (Group Walk-Turn) walked the route in a normal manner facing forward, and the second group (Group Backward-Turn) walked the route backward. Subjects in the third group (Group WalkNo Turn) maintained a constant orientation to the routes as they walked. This required them to walk sideways (or diagonally), as well as forward and backward. The main question to be tested was whether, when asked to make judgments while either aligned or contra-aligned with their initial experience at that location, subjects would be differentially biased to recall the route information in a single orientation as a function of their initial learning experience of the routes. If their knowledge of the routes was orientation specific, they would be expected to show alignment effects, so that judgments would be more accurate on aligned trials than on contra-aligned trials. In contrast, if the route information was coded in an orientation-free manner, no alignment effects would be expected.

On the basis of past work (Evans \& Pezdek, 1980; Presson \& Hazelrigg, 1984), Group Walk-Turn, which walked normally and had multiple orientations during learning, was not expected to show alignment effects. To test for the importance of multiple orientations, the key group (Group Walk-No Turn) was the one that walked by maintaining a single orientation to the route during learning. If multiple orientations while learning the route kinesthetically are necessary to obtain orientation-free coding, then Group Walk-No Turn would be expected to code the route information in an orientation-specific way and to demonstrate strong alignment effects. However, if spatial information obtained from kinesthetic experience is generally stored in an orientation-free manner regardless of the number of orientations during learning, then neither Group Walk-No Turn nor Group Walk-Turn would be expected to show alignment effects.

A difference between Groups Walk-Turn and WalkNo Turn might be due either to the number of orientations during learning or to the unusual walking style for Group Walk-No Turn. In order to control for any possible effects of the unusual walking style required of Group Walk-No Turn, a third group (Group BackwardTurn) was later included as a nonequivalent control group (Cook \& Campbell, 1979). Group Backward-Turn walked the routes backward and turned as appropriate for each segment of the route, thereby experiencing the route in multiple orientations. If the predicted difference in alignment effects between Groups Walk-Turn and WalkNo Turn occurred, Group Backward-Turn would allow us to identify the critical factor unambiguously. If the unusual walking style is the critical factor in determining alignment effects, then Group Backward-Turn and Group Walk-No Turn would be expected to show alignment effects. In contrast, if multiple orientations are the critical factor, then alignment effects would be obtained only for Group Walk-No Turn. Both Groups Walk-Turn and Backward-Turn were expected to show no alignment effects.

In addition to assessing alignment effects in terms of accuracy of directional judgment, the current study also examined response times for aligned and contra-aligned judgments. It may be that subjects who learn spatial information kinesthetically are biased toward recalling the information in a specific orientation, but that the bias does not affect the accuracy of subsequent location judgments. Such bias might show up in longer response times for contra-aligned than for aligned trials, even if the two trial types are of equivalent accuracy. If the representations are available in a truly orientation-free manner, however, then response time (as well as accuracy) should be equivalent for both aligned and contra-aligned trials.

\section{METHOD}

\section{Subjects}

Thirty-six undergraduate student volunteers (18 males and $18 \mathrm{fe}$ males) participated. Twenty-four (Groups Walk-Turn and WalkNo Turn) were students attending the University of Missouri at Columbia, and 12 (Group Backward-Turn) were students at Arizona State University. Participation was a requirement in their psychology classes.

\section{Materials}

Eight routes were designed as stimuli. Each route had three linear segments connecting four locations, and the length of each route segment varied from 100 to $300 \mathrm{~cm}$. The angles between segments were $90^{\circ}-90^{\circ}, 135^{\circ}-45^{\circ}$, or $45^{\circ}-135^{\circ}$. All routes were U-shaped, with the first and third segments parallel, and were laid out on the floor of a large room.

When tested for their route knowledge, the subjects made their directional judgments using a circular dial $(30-\mathrm{cm}$ diameter) with a pointer that could be freely rotated around the dial. The dial was hand-held, with notches at $0^{\circ}, 90^{\circ}, 180^{\circ}$, and $270^{\circ}$ to provide reference points for the blindfolded subjects. The dial was marked in $5^{\circ}$ increments from $0^{\circ}$ to $355^{\circ}$ clockwise from straight ahead, and responses were recorded to the nearest $5^{\circ}$. Response times were measured to the nearest $.1 \mathrm{sec}$ with a hand-held stopwatch.

\section{Procedure}

Each subject learned eight routes in one of three walking conditions (Conditions Walk-Turn, Backward-Turn, and Walk-No Turn). In all conditions, the subject was blindfolded, seated in a chair on rollers, and moved to a corner of a large room. The blindfold was removed while the basic task was explained and the response pointer was demonstrated. The subject was then reblindfolded and moved to the position for learning the first route. He/she remained blindfolded throughout the entire testing session and was seated except when walking on each route and making judgments.

The subjects walked each route three times. Each was positioned (in the chair) at the beginning of a route and told that they were at Location 1. The subject then stood and was led over the route by the experimenter, who stopped the subject and announced each location as it was encountered. At the end of each route, the subject was reseated in the chair (which had been rolled along behind the subject as he/she walked) and was wheeled in a meandering path back to Location 1 for his/her next walk over the route.

The subjects in Condition Walk-Turn walked the routes normally. They were turned at each location by the experimenter so that they always walked straight ahead. The subjects in Condition BackwardTurn walked the routes backward and were turned at each location 
so that they continued to walk the same way through the route. The subjects in Condition Walk-No Turn maintained a constant orientation to the route as they walked. This required them to walk sideways (or diagonally), as well as backward and forward, over the three segments of the route. For half of the routes, Group WalkNo Turn began walking forward and, because the first and last segments were parallel, ended by walking backward. On the other routes, the subjects began walking backward and ended walking forward.

After the subjects had learned a route by any method, they were asked to make two judgments about the route, one from Location 1 and one from Location $4 .{ }^{1}$ While still blindfolded, the subjects were rolled to Location 1 or Location 4 , were oriented with respect to their location and heading (e.g., "You are at Location 1, and Location 2 is directly in front of you'), and were asked to direct the pointer to another location on the route ("Point to where Location 3 is"). Both the direction of the pointer and the response time were recorded for each judgment. Response times were measured from the time the target was named until a subject began directing the pointer. After the first judgment, the subjects were rolled to Location 2 and asked to make another judgment. No feedback was provided during the testing session, and the subjects were debriefed after their participation.

Each of the subjects made 16 judgments ( 2 for each route). Across trials, 8 of these judgments were made from an aligned position and 8 from contra-aligned positions, although judgments could be both aligned, both contra-aligned, or one of each for any particular route. Alignment was determined by the direction the subjects faced when they arrived at that location on the route: An aligned judgment was made with the subjects facing the same direction as when they had learned the route; a contra-aligned judgment was made with the subjects facing in the opposite direction from which they had learned that location. For example, if a subject arrived at Location 4 by walking backward (e.g., facing Location 3), an aligned judgment would place the subject "at Location 4, with Location 3 directly in front of you." For a contra-aligned judgment from the same location, a subject was told, "You are at Location 4 and Location 3 is directly behind you."

\section{RESULTS}

\section{Accuracy of Pointing}

The angles of absolute error for each type of pointing judgment were analyzed in a 3 (learning condition: WalkTurn, Walk-No Turn, and Backward-Turn) $\times 2$ (alignment: aligned or contra-aligned) $\times 2$ (subjects' orientation: facing on or off the route) $\times 2$ (direction of pointing: in front of or behind the subject) mixed-design ANOVA. ${ }^{2}$ There was no overall main effect for learning condition: Mean degrees of error were $45.1^{\circ}, 41.9^{\circ}$, and $31.6^{\circ}$ for Groups Walk-Turn, Walk-No Turn, and Backward-Turn, respectively. This accuracy was well above chance expectancy (chance $=90^{\circ}$, because errors could range from $0^{\circ}$ to a maximum of $180^{\circ}$ ) for each group (binomial tests, $p<.01$ ).

The major question concerned alignment effects within each learning group. There was a main effect of alignment $[F(1,33)=10.41, p<.003]$, which was qualified by the predicted alignment condition interaction (see Figure 1) $[F(2,33)=7.08, p<.003]$. Subsequent tests for simple effects indicated a reliable difference between aligned versus contra-aligned trials only for Group WalkNo Turn $[F(1,33)=21.91, p<.0001]$, with no reliable

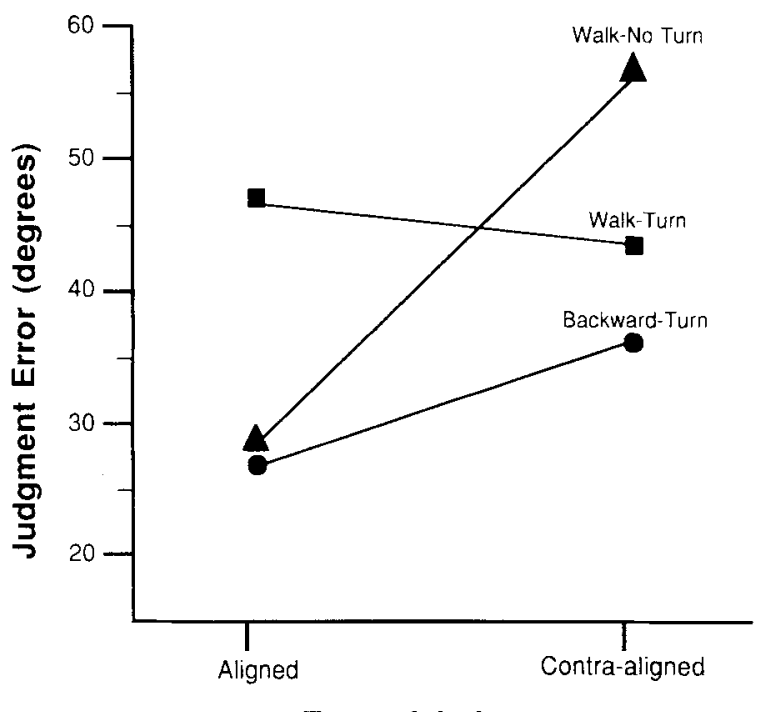

Type of Judgment

Figure 1. Mean directional judgment error (in degrees) collapsed across trial type for Groups Walk-Turn, Backward-Turn, and WalkNo Turn.

differences between aligned versus contra-aligned judgments for Group Walk-Turn $(F<1)$ or Group BackwardTurn $[F(1,33)=2.30, p<.13]$. Group Walk-No Turn subjects' average error increased $28.4^{\circ}$ from an average of $27.7^{\circ}$ for aligned trials to $56.2^{\circ}$ for contra-aligned trials. In contrast, the difference between aligned and contra-aligned trials for Group Walk-Turn subjects was $-3.7^{\circ}$ (mean aligned trials $=46.9 ;$ mean contra-aligned trials $=43.2$ ) and the difference for Group BackwardTurn subjects was $9.2^{\circ}$ (mean aligned trials $=27.0$; mean contra-aligned trials $=36.2$ ).

In addition to the predicted alignment $\times$ condition interaction, the interaction of subjects' orientation and direction of pointing $[F(1,33)=11.42, p<.01]$ and alignment and direction of pointing $[F(2,33)=4.17, p<.05]$ indicated that the subjects were more accurate when pointing into the bulk of the route than when pointing away from it and were more accurate on contra-aligned trials when pointing in front of (rather than behind) themselves.

These data clearly show the importance of multiple orientations in achieving orientation-free spatial representations. Group Walk-No Turn showed alignment effects, whereas the other two groups did not.

To look more closely at the patterns within the accuracy data, we first examined over- and underestimates in directional judgments. The distributions of these (signed) errors were fully symmetric for both aligned and contraaligned judgments in all three groups. Because no systematic bias occurred, only absolute errors were examined further. The distributions of absolute error for aligned and contra-aligned judgments were unimodal and indistinguishable for both Groups Walk-Turn and BackwardTurn. Consistent with the obtained alignment effects, Group Walk-No Turn had different distributions for aligned and contra-aligned judgments (see Figure 2). The 


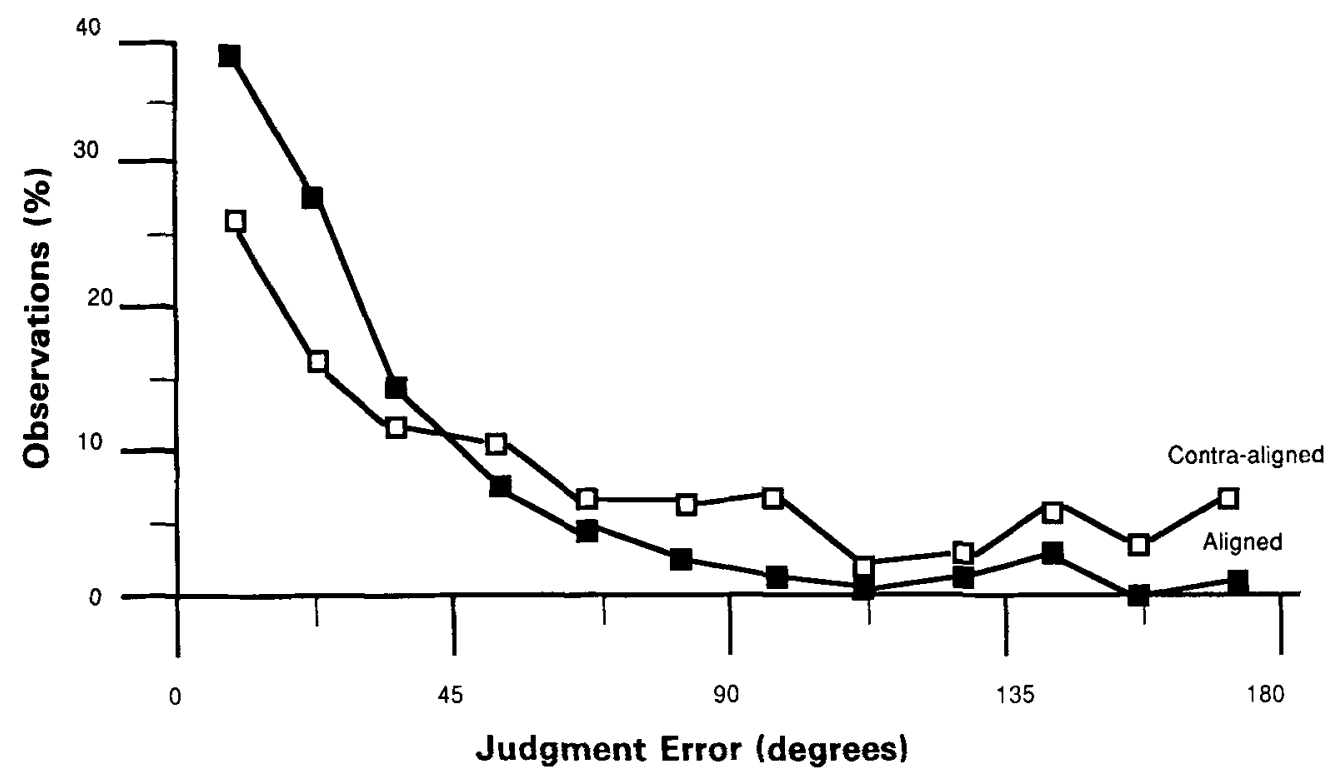

Figure 2. Distribution of directional (absolute) errors for aligned and contra-aligned judgments for Group Walk-No Turn.

distributions differed across the entire range of potential errors, and the distribution of contra-aligned trials was not clearly bimodal, which indicates that the subjects were not simply failing to adjust for contra-aligned judgments. (Such a failure would result in contra-aligned errors clustering around $180^{\circ}$.)

\section{Response Times}

The data for response times essentially mirrored the findings for absolute error. A condition $\times$ alignment interaction $[F(2,33)=5.51, p<.01]$ indicated that response times for contra-aligned judgments were significantly slower than those for aligned judgments for the Group Walk-No Turn subjects only [test of simple effects, $F(1,33)=26.90, p<.0001]$. These subjects were $5.35 \mathrm{sec}$ slower for contra-aligned judgments $(12.71 \mathrm{sec})$ than for aligned judgments $(7.36 \mathrm{sec})$. In contrast, aligned and contra-aligned judgments were equally fast for Groups Walk-Turn and Backward-Turn [tests of simple effects, $F(1,33)=2.48, p<.12$, and $F(1,33)<1$, respectively]. For Group Walk-Turn, the mean responses were $9.37 \mathrm{sec}$ for contra-aligned judgments and $7.74 \mathrm{sec}$ for aligned judgments, and for Group Backward-Turn, the mean responses were $3.82 \mathrm{sec}$ and $3.01 \mathrm{sec}$ for contraaligned and aligned judgments, respectively.

Overall, the subjects were faster when pointing in front of $(6.91 \mathrm{sec})$ than when pointing behind $(7.76 \mathrm{sec})$ themselves $[F(1,33)=6.33, p<.02]$, and were faster when pointing toward the bulk of the route $(6.69 \mathrm{sec})$ than when pointing away from it $(7.98 \mathrm{sec})[F(1,33)=7.13$, $p<.01]$.

\section{DISCUSSION}

The current study was designed to identify how turning while learning a route kinesthetically (blindfolded walking) affected the representation of the route information. In particular, the question was whether turning would affect the orientation specificity of the spatial representation, as indexed by alignment effects in later judgments. The results were clear. Experiencing multiple orientations by turning while walking was critical to whether the subjects showed alignment effects. When the blindfolded subjects walked and experienced multiple orientations, directional judgments to other locations on the route were equally accurate (and equally fast) regardless of whether those judgments were aligned or contraaligned with initial learning. In contrast, when the routes were learned while the subjects walked in a constant orientation (without turning), the subsequent judgments showed marked alignment effects, both for accuracy and response times.

The difference in the alignment effects between Group Walk-No Turn and Groups Walk-Turn and BackwardTurn indicates that the route information was treated in distinct ways when kinesthetically learned in single versus multiple orientations. The absence of any alignment effects for Groups Walk-Turn and Backward-Turn indicates that the route information in these conditions was equally accessible in any orientation. No alignment effects were found when the route was learned by normal (forwardfacing) walking or when it was learned by backward walking. Furthermore, no alignment effects were found for accuracy or for response times, which suggests that the route representations were truly orientation free.

In contrast, for Group Walk-No Turn, route knowledge was tied to the specific learned orientation. The information was retrieved in that specific orientation, and additional time and effort were necessary to readjust the information to align it with the judgment. This process of adjustment on contra-aligned judgments led both to longer response times and to decrements in accuracy. An exami- 
nation of the distributions of pointing responses showed that the judgments on the contra-aligned trials did not have a clear bimodal distribution with some accurate (no errors) and some very inaccurate (clustered around $180^{\circ}$ incorrect) responses. That type of bimodal distribution had been shown previously for representations learned visually from maps (Levine et al., 1982; Presson \& Hazelrigg, 1984). Levine et al. interpreted this bimodality as occurring when subjects failed to adjust their representations of the visually learned map to the demands of the task in contra-aligned judgments. The fact that the distribution of errors for the Group Walk-No Turn contra-aligned judgments was not similarly bimodal suggests that the subjects in that group did recognize the need to adjust their spatial representations for contra-aligned judgments, but were only partially successful in doing so.

Thus, the current findings support the importance of multiple orientations for orientation-free, flexible spatial representations in kinesthetic learning. Kinesthetic learning, per se, does not always lead to orientation-free spatial representations-multiple orientations during learning are also necessary. This finding is consistent with Evans and Pezdek's (1980) suggestion that multiple vantage points might be a critical factor that differentiates sighted navigation (which does not have alignment effects) from map learning (which does). However, it is important not to generalize the current findings beyond kinesthetic spatial learning, because multiple orientations are not necessary for spatial learning to be represented in an orientation-free manner in other modalities.

In particular, we have shown previously (Presson \& Hazelrigg, 1984) that, for visual spatial learning, multiple vantage points are not necessary for orientation-free coding. In that study, a map-learning group was contrasted with a group that learned routes by looking directly at them on the floor from a single vantage point. Although both groups viewed the array from only a single vantage point, only the map-learning group showed alignment effects in later judgments. The direct-look group did not. We suggested that a critical distinction between the group that showed alignment effects (map learning) and the groups that did not (the direct-look group and a normal kinesthetic learning group) was that the map group learned the spatial information in an indirect, symbolic form (secondary spatial learning). In contrast, the look and map groups learned the routes from a more direct, primary spatial experience.

The current data question whether the distinction between primary and secondary forms of spatial learning is the only factor that governs the orientation specificity of spatial representations. At least within kinesthetic learning, multiple orientations are a critical factor. In the current study, all groups had a direct, primary experience of the route (although Groups Backward-Turn and WalkNo Turn had an unusual one), and it was the multiple versus single orientations during learning that determined the orientation specificity of the representations.

The specific aspects of the spatial learning experience that lead people to represent spatial information in either orientation-specific or orientation-free form may, in fact, differ across spatial modalities. For kinesthetic learning, it is clear that multiple orientations are critical to the orientation specificity of coding. For visual forms of spatial learning, whether the experience is primary or secondary is an important factor (Presson \& Hazelrigg, 1984). The further identification of the conditions under which people code spatial information in different ways remains a challenge to a full understanding of spatial representation.

\section{REFERENCES}

COOK, T. D., \& CAMPBELl, D. T. (1979). Quasi-experimentation: De sign and analysis issues for field settings. Chicago: Rand McNally. Evans, G., \& PEZDEK, K. (1980). Cognitive mapping: Knowledge of real-world distance and location information. Joumal of Experimental Psychology: Human Learning \& Memory, 6, 13-24.

JoliCOEUR, P., \& KossLYN, S. (1983). Coordinate systems in the longterm memory representations of three-dimensional shapes. Cognitive Psychology, 15, 301-345.

Levine, M., Jankovic, I., \& PaliJ, M. (1982). Principles of spatial problem solving. Journal of Experimental Psychology: General, 111, 157-175.

LIBEN, L. S. (1981). Spatial representation and behavior: Multiple perspectives. In L. S. Liben, A. H. Patterson, \& N. Newcombe (Eds.), Spatial representation across the life-span (pp. 3-36). New York: Academic Press.

MANDLER, J. (1983). Representation. In J. H. Flavell \& E. Markman (Eds.), Handbook of child psychology: Vol. 3. Cognitive development (pp. 420-494). New York: Wiley.

Presson, C. C., \& Hazelrigg, M. D. (1984). Building of spatial representations through primary and secondary learning. Journal of Experimental Psychology: Learning, Memory, \& Cognition, 10, 716-722.

ThORNDYKE, P. W., \& HAYES-Roth, B. (1982). Differences in spatial knowledge acquired from maps and navigation. Cognitive Psychology, 14, 560-581

\section{NOTES}

1. Only Locations 1 and 4 were used as judgment points so that in all cases subjects would have only one orientation during learning while at these test locations. This allowed for unambiguous assignment of any judgment as aligned or contra-aligned. The subjects pointed to Locations 1 and 2 from Location 4; from Location 1, the subjects pointed to Locations 3 and 4.

2. Group Backward-Turn was included in this analysis as a nonequivalent control group (Cook \& Campbell, 1979). Because our hypotheses required only tests of the presence or absence of alignment effects, any differences between Group Backward-Turn and the other two groups as a function of specific population, time, or location are assumed to be irrelevant to the hypotheses tested.

(Manuscript received January 16, 1985; revision accepted for publication September 29, 1986.) 